\title{
- Introduction to the Inaugural Issue: A Special Issue on Interdisciplinary Work at the Intersection of Systems and Human Sciences
}

\author{
Leila Takayama, \\ Willow Garage \\ and \\ Brian Scassellati \\ Department of Computer Science, Yale University
}

The intertwining of people and robots is at the very heart of human-robot interaction. The design of effective human-robot interactions depends not only upon an elegant and thorough engineering design for the robot's behavior, but also a clear and insightful understanding of how the human user is likely to behave toward the robot. This presents a challenge and an opportunity for engaging robotics systems research with the cognitive, behavioral, and social sciences. Without a deeper understanding of human user contexts, real user needs, and an ability to evaluate a robotic system's performance in terms of user needs, robotics runs the risk of inventing technologies for the sake of the technologies themselves. Without a thorough understanding of the state-of-the-art in robotics, user research runs the risk of generating superficial or irrelevant recommendations for robotic system design.

To help understand and address the risks and opportunities for collaboration between robotics and the human-centered sciences, we ran a workshop at the Robotics Science and Systems (RSS) conference in 2011 at the University of Southern California (RSS Workshop, 2012). The workshop brought together members of both communities to more openly discuss, debate, and ultimately learn from one another. This workshop inspired this inaugural special issue of the Journal of HRI, highlighting research that demonstrates taking both understanding people and understanding robotic systems seriously.

We received an overwhelming response to our initial request for contributions for this inaugural issue. Many papers passed a multiple-stage review process and received lengthy and detailed reviews from senior experts in the field. From these candidates, we selected six papers that best exemplified the duality of both robot systems-building and careful consideration of the human-centered issues surrounding these interactions.

The first three articles demonstrate the strengths of combining both systems sciences and user studies, including clinical studies and controlled laboratory experiments. First, Chao and Thomaz leverage turn-taking interactions between people to develop a multimodal collaboration system that is implemented on a humanoid robot; they offer the results of user studies that showed how a robot's action interruptions improve task efficiency. Second, Kim, et al. share their findings from ten years of collaborating with clinical researchers to address the needs of individuals with autism, including empirical results of a human-robot interaction study that demonstrates improvements in human-human social interaction. Third, Feil-Seifer and Matarić report upon their development of socially-aware goal-oriented navigation behaviors for robots as well as their user studies with children with autism spectrum disorders and with neurotypical adults. These authors

\footnotetext{
Authors retain copyright and grant the Journal of Human-Robot Interaction right of first publication with the work simultaneously licensed under a Creative Commons Attribution License that allows others to share the work with an acknowledgement of the work's authorship and initial publication in this journal.
}

Journal of Human-Robot Interaction, Vol. 1, No. 1, 2012, Pages 2-3. DOI 10.5898/JHRI/1.1.Takayama 
demonstrate how autonomous robotic social intelligence can improve both task performance and interpersonal engagement for children with ASD.

The subsequent three articles are examples of even more diverse combinations of systems and user studies, including field studies, design explorations, and research methods in HRI. The fourth article, by Tanaka and Matsuzoe, presents the lessons learned from putting a care-receiving robot into an elementary school classroom; these lessons emphasize how such robots can support learning outcomes for children. Fifth, Mast, et al. report upon their user-centered iterative design process for developing a service robot that physically assists older adults in their homes; they identify valuable services for robots to provide, insights into robotic tele-assistance, and three different user roles that need to be supported by these service robots. Finally, Riek reviews dozens of Wizard-of-Oz experiments in order to provide guidance on how to most effectively use these methods in HRI research and how to report upon the results of these types of user studies. This work discusses the place of Wizard-of-Oz studies in HRI, addressing the needs of real robots "in the wild" amongst real end-users, turning user study results into user interface requirements for robotic system development.

Taken together, these papers demonstrate some of the trends that have become evident in human-robot interaction in the past few years. These articles draw from a wide range of disciplines (including psychology, education, clinical practice, sociology, and anthropology) and make use of methodologies that are equally broad (including user studies, clinical evaluations, focus groups, ethnographic surveys, and engineering evaluations). Similarly, there is an extremely wide range of robots employed in these studies - custom-built robots to off-the-shelf commercial systems, humanoid robots to non-anthropomorphic robots, autonomous control systems to remotely operated systems. This diversity of both platforms and disciplinary connections reflects the youth and diversity of the current HRI research environment. While the breadth of coverage of these six papers is considerable, it obviously is not completely representative of the landscape of HRI studies present today. Notably missing are papers that cover non-social robotic systems, long-term ethnographic methods, and application domains outside of assistive technology.

If one overarching lesson can be taken from this collection of papers, it is that the collaborations between human-centered researchers and robotics researchers has borrowed heavily from the human-centered disciplines to establish rigorous and validated evaluation mechanisms. Much more today than even a few years ago, HRI papers have begun to benefit tremendously from a rigorous evaluation using methods drawn from social and behavioral sciences. At the same time, the unique aspect of HRI research is that these methods must be adapted to the particular challenges and opportunities that robotics research presents. It is our hope as editors that these papers in particular might serve as guideposts to encourage future efforts in these areas to follow and extend these rigorous evaluation methods.

Many thanks go to the generous manuscript submitters, reviewers, and senior editors, who made this inaugural special issue possible. We hope that these articles will be as insightful and useful for you as they are for us.

\section{References}

RSS Workshop (2011). RSS 2011 Workshop on Human-Robot Interaction: Perspectives and Contributions to Robotics from the Human Sciences. Organized by L. Takayama, M. Mataric', O C. Jenkins, H. Yanco, and B. Scassellati. Last access date 25 July 2012. http://hri.willowgarage.com/workshops/RSS2011/RSS_2011_Human-Robot_Interaction.html

Guest editors' contact information:

Leila Takayama, Willow Garage, Inc., Menlo Park, California, USA. Email: takayama@willowgarage.com.

Brian Scassellati, Department of Computer Science, Yale University, New Haven, Connecticut, USA. Email: brian.scassellati@yale.edu. 